\title{
Attention to detail and a permissive set-up: crucial for an effective TURBT
}

\section{Paramananthan Mariappan (B)}

\begin{abstract}
An effective transurethral resection of bladder tumour (TURBT) is essential to achieve safe tumour clearance and to determine the biological potential of the cancer. Attention to surgical detail within a set-up that embraces evidence-based practice, training, quality standards, prospective audit and feedback will facilitate these objectives.
\end{abstract}

Refers to Das, A. et al. Surgeon scorecards improve muscle sampling on transurethral resection of bladder tumor and recurrence outcomes in patients with nonmuscle invasive bladder cancer. J. Urol. 205, 693-700 (2021).

The aspiration of surgeons performing transurethral resection of bladder tumour (TURBT) is the conflation of bladder preservation along with the objective triad of cancer clearance, acquisition of clinical and histopathological information to determine the biological potential of the tumour, and the safe performance of the procedure (including reducing the risk of potential tumour dispersion). Reliable metrics of effective tumour clearance, that is, the quality of the resection in non-muscle invasive bladder cancer (NMIBC), are absence of recurrence at the first check cystoscopy and the absence of residual cancer at early re-look TURBT (in high-grade NMIBC). These end points, which are highly predictive of subsequent cancer recurrence and progression, are unfortunately reliant on a future examination; thus, aside from the documentation of 'complete clearance' in the operation note by the surgeon, sampling of detrusor muscle, as determined on histopathological assessment, can serve as an objective surrogate of resection quality.

Seeking to improve the quality of TURBT in their institution, Das et al. ${ }^{1}$ evaluated the utility of a scorecard in providing feedback to surgeons with regard to detrusor muscle sampling at TURBT ${ }^{1}$. An initial retrospective interrogation of their database provided individual surgeon stage-stratified detrusor muscle sampling rates over a 10 -year period. In 2017 , these data were presented to the 11 surgeons who performed at least ten TURBTs per year, as a bespoke scorecard featuring the individual's detrusor muscle sampling rates in $\mathrm{T} 1$ cancers over time, along with a graphical comparison with the other $10 \mathrm{col}-$ leagues. In the 12 months following receipt of the scorecard, a further 373 TURBTs were undertaken, and analysis revealed a significant (pooled) increase in detrusor muscle sampling during TURBT when compared with data from procedures performed before receipt of the scorecard ( $36 \%$ ( 1,250 of 3,488 procedures) to $54 \%$ (202 of 373 ) for all TURBTs, $P=0.001$; $47 \%$ ( 183 of 390 procedures) to $72 \%$ (42 of 58 procedures) in T1 cancers, $P<0.001$ ).

\section{TURBT cannot be an} esoteric procedure performed by only a few surgeons

After exclusions, follow-up data were available for 591 patients (479 who underwent TURBT before scorecard distribution; 112 after scorecard receipt). Recurrence seemed to be reduced between the two patient cohorts from censored data available at 36 months (34\% versus $20 \%, P<0.001$ ). However, given the limitations of the study, particularly absence of a control group or propensity-score matching, the modest sample size and potential selection bias, their conclusion that 'creation and distribution of individual scorecards improved detrusor muscle sampling' is easy to repudiate.
Nonetheless, the researchers must be congratulated for adding to the evidence base highlighting the importance of audit and feedback in improving the effectiveness of TURBT.

At the more aggressive end of the NMIBC spectrum are pT1 and high-grade cancers; the absence of detrusor muscle in the initial resection, coupled with not performing a second re-look resection, are deemed harbingers of poorer oncological outcomes in patients with these tumours ${ }^{2}$. Conversely, the value of sampling detrusor muscle in low-grade, non-invasive cancers is somewhat debateable. However, at initial diagnosis, visual distinction between high-grade and low-grade cancer is challenging, even for experienced surgeons ${ }^{3}$. Thus, unless faced with a thin-walled bladder or very small papillary lesions, attempting to sample the detrusor muscle for pathological staging would be a logical approach in all initial TURBT procedures.

The challenging issue of staging accuracy could also be augmented preoperatively by multiparametric MRI using Vesical Imaging Reporting and Data System (VI-RADS). Recent evidence suggests that VI-RADS has the potential to distinguish between muscleinvasive bladder cancer and NMIBC and identifies those with high-grade cancer most likely to benefit from an early re-look TURBT ${ }^{4}$. It is my opinion that in time, supported by machine learning, such advancements might negate routine re-look TURBT. However, until multiparametric MRI is used routinely in clinical practice, pathological staging will be reliant on performing a timely and effective TURBT.

The crucible of COVID-19 has further highlighted an unprecedented need for efficiency and effectiveness in health care. Bladder cancer services, and in particular TURBT services, must be effective, and the Getting It Right First Time (GIRFT) NHS quality improvement programme in England, for example, is one such national initiative that could facilitate reducing reliance on the second re-look TURBT (as this is, essentially, an admission that the procedure was not effective the first time). Achieving adequate resection at the initial TURBT requires the appropriate operative set-up and attention to detail during the procedure. Although centralization of specialist services is more likely to achieve these principles, for pragmatic reasons, we cannot afford the standard TURBT to be performed only by a select few surgeons. 


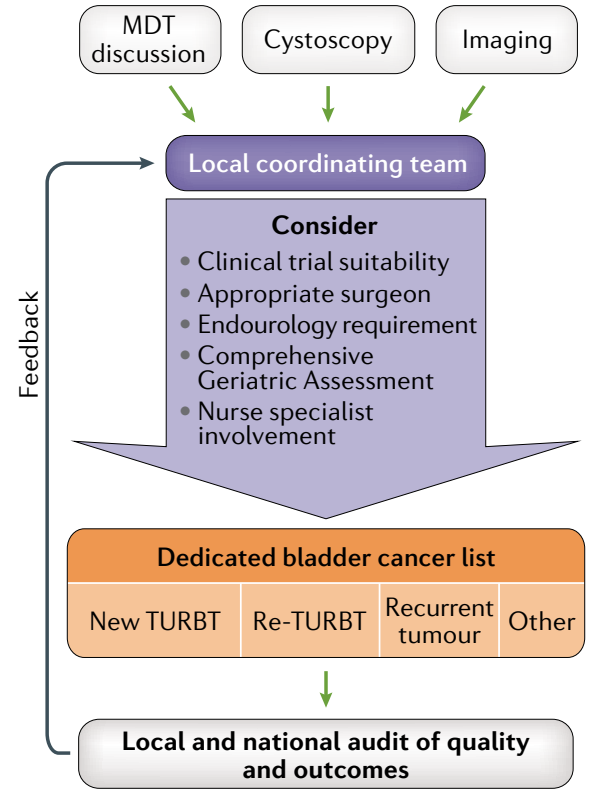

Fig. 1 | Principles and processes in TURBT.

Before transurethral resection of bladder tumour (TURBT), the local coordinating team should triage the patient to the most appropriate operating list, taking into consideration the need for specialist intervention, ensuring good communication with the patient throughout. Multidisciplinary team (MDT) and, in some patients, Comprehensive Geriatric Assessment (CGA) input are often required before TURBT. All procedures should ideally be performed on a dedicated list with ring-fenced slots with the availability of appropriate optical enhancements and post-operative intravesical chemotherapy instillation where appropriate. Prospective audit of data and feedback of quality and outcomes of TURBT should be undertaken by all centres.

Embracing the Donabedian three-component principles of structure, process and outcomes in the evaluation of health-care quality, the TURBT service delivery is more likely to facilitate effectiveness of the procedure and patient-centred care by incorporating a model that has dedicated TURBT operating lists with adequate operating time and expertise, coordinates surgery through a process of patient triage and takes several aspects of the patient and their disease into account, including the use of Comprehensive Geriatric Assessment (CGA), which help to guide the appropriateness of a procedure (or perhaps lead to the adoption of a more supportive approach when necessitated by co-morbidities). The model should also incorporate good communication between surgeon and patient with the support of a specialist nurse and access to the recruitment of patients into clinical trials that explore recent developments, and there should be encouragement of evidence-based practice followed by close prospective audit and feedback ${ }^{5}$ (FIG. 1).
Quality indicators can help define standards and introduce evidence-based metrics along the TURBT pathway ${ }^{6}$. National quality improvement programmes in bladder cancer that facilitate variance-free and evidence-based care enable better patient outcomes, improve patient experience, and can lead to more effective predictions of the disease course. An example of this is the Scottish bladder cancer Quality Performance Indicator programme, wherein inherent governance processes, incorporating mandatory prospective audit, annual feedback and health-board accountability, are central to the emerging improvement in clinical outcomes for patients; data have demonstrated consistent detrusor muscle sampling rates of approximately $80 \%$ with commensurate risk of under-staging in only $3 \%$ of patients ${ }^{5}$.

Although the introduction of reference standards and performance-based feedback have the potential to improve outcomes, a challenge might be the adherence to evidencebased guidelines in bladder cancer ${ }^{7}$. For instance, the adherence to both the American and European Association of Urology guidelines recommending a single post-TURBT instillation of chemotherapy was only $43 \%$ in both Europe and Australia and $0.5 \%$ in North America ${ }^{7}$. Clinicians' acceptance of guidelines has been proposed as one of the barriers to evidence-based practice ${ }^{8}$. To aid management of behavioural divergence in practice, innovative approaches (such as use of a 4-step process: initially identify the gap between evidence and practice; highlight barriers to adoption of appropriate evidence; identify changes to overcome such barriers and enhance enablers; and measure outcomes following behavioural change) are necessary to introduce theory-driven behavioural change to support acceptance of the evidence and translate this into clinical practice ${ }^{8}$. Such a methodical evaluation of factors that obstruct and facilitate the implementation of evidencebased practice might help improve the utilization of a single post-TURBT instillation of chemotherapy ${ }^{9}$. Despite the evidence that composite measurements of surgical quality can potentially influence clinical outcomes ${ }^{6}$, adopting a pragmatic approach to developing benchmarks and practice standards is more likely to be successful ${ }^{10}$.

Training is a vital element in this process. Sadly, TURBT is often considered prosaic, with training in TURBT becoming an unintentional victim of the lure of other surgery using more technical and expensive equipment. With the growing emphasis on close supervision and use of optical enhancements, the somewhat autodidactic method of learning TURBT is largely outdated and a more hands-on approach in training future surgeons, combined with simulation, is warranted. Apart from being enforcers of attention to detail, trainers need to also embrace pedagogical skills not only to improve trainees' technical ability but also to passionately inspire interest in this field.

Demand within health-care (especially, public-funded) systems means that TURBT cannot be an esoteric procedure performed by only a few surgeons. For the procedure to have optimal clinical outcomes, adherence to evidence-based guidelines with demonstration of the achievement of appropriate standards is necessary. A system that embraces audit, feedback and training, preferably within some framework of governance, should be encouraged. Proclivity for attention to detail within such a system will almost certainly encourage excellence in patient care.

Paramananthan Mariappan (iD ${ }^{1,2}$

Edinburgh Bladder Cancer Surgery, Department of Urology, Western General Hospital, Edinburgh, UK.

${ }^{2}$ University of Edinburgh, Edinburgh, UK.

e-mail: param.mariappan@nhslothian.scot.nhs.uk https://oi.org/10.1038/s41585-021-00441-9

1. Das, A. et al. Surgeon scorecards improve muscle sampling on transurethral resection of bladder tumor and recurrence outcomes in patients with nonmuscle invasive bladder cancer. J. Urol. 205, 693-700 (2021)

2. Gontero, P. et al. The impact of re-transurethral resection on clinical outcomes in a large multicentre cohort of patients with T1 high-grade/grade 3 bladder cancer treated with bacille Calmette-Guérin. BJU Int. 118, 44-52 (2016).

3. Mariappan, P. et al. Predicting grade and stage at cystoscopy in newly presenting bladder cancers-a prospective double-blind clinical study. Urology 109 134-139 (2017).

4. Del Giudice, F. et al. Prospective assessment of Vesical Imaging Reporting and Data System (VI-RADS) and its clinical impact on the management of high-risk non-muscle-invasive bladder cancer patients candidate for repeated transurethral resection. Eur. Urol. 77 , 101-109 (2020)

5. Mariappan, P. et al. Enhanced quality and effectiveness of transurethral resection of bladder tumour in nonmuscle-invasive bladder cancer: a multicentre realworld experience from scotland's quality performance indicators programme. Eur. Urol. 78, 520-530 (2020).

6. Leow, J. J. et al. Quality indicators for bladder cancer services: a collaborative review. Eur. Urol. 78, 43-59 (2020).

7. van Rhijn, B. W. G. \& Burger, M. Bladder cancer: low adherence to guidelines in non-muscle-invasive disease. Nat. Rev. Urol. 13, 570-571 (2016).

8. French, S. D. et al. Developing theory-informed behaviour change interventions to implement evidence into practice: a systematic approach using the Theoretical Domains Framework. Implement Sci. 7, 38 (2012).

9. Dunsmore, J. et al. What influences adherence to guidance for post-operative instillation of intravesical chemotherapy to bladder cancer patients? BJU Int https://doi.org/10.1111/bju. 15336 (2021).

10. Atherton, H. et al. Evidence scan: the impact of performance targets within the NHS and internationally. The Health Foundation http://www.health.org.uk/sites/ default/files/ThelmpactOfPerformanceTargetsWithin TheNHSAndInternationally_0.pdf (2015).

\section{Competing interests}

The author declares no competing interests.

\section{RELATED LINKS}

Getting It Right First Time: https://www.gettingitrightfirsttime. co.uk/ 